\title{
A Diagnostic Algorithm for Mitochondrial Disorders in Estonian Children
}

\author{
K. Joost ${ }^{\text {a, c }}$ R.J. Rodenburg ${ }^{g} \quad$ A. Piirsoo ${ }^{d}$ L. van den Heuvel ${ }^{g}$ R. Žordania ${ }^{a}$ \\ H. Põder ${ }^{f} \quad$ I. Talvik ${ }^{\text {b, e }} \quad$ K. Kilk ${ }^{c}$ U. Soomets ${ }^{c}$ K. Õunap ${ }^{\text {a, e }}$ \\ ${ }^{a}$ Department of Genetics, United Laboratories, and b Children's Clinic, Tartu University Hospital, \\ 'The Centre of Excellence for Translational Medicine, ${ }^{d}$ Department of General and Molecular Pathology, and \\ eDepartment of Pediatrics, University of Tartu, Tartu, and fTallinn Children's Hospital, Tallinn, Estonia; \\ ${ }^{9}$ Nijmegen Centre for Mitochondrial Disorders, Radboud University Nijmegen Medical Centre, \\ Nijmegen, The Netherlands
}

\section{Key Words}

Diagnostic criteria $\cdot$ Lactate $\cdot$ Mitochondrial disease $\cdot$

Prevalence

\begin{abstract}
Mitochondrial disorders are a heterogeneous group of disorders affecting energy production of the body. Different consensus diagnostic criteria for mitochondrial disorders in childhood are available - Wolfson, Nijmegen and modified Walker criteria. Due to the extreme complexity of mitochondrial disorders in children, we decided to develop a diagnostic algorithm, applicable in clinical practice in Estonia, in order to identify patients with mitochondrial disorders among pediatric neonatology and neurology patients. Additionally, it was aimed to evaluate the live-birth prevalence of mitochondrial disorders in childhood. During the study period (2003-2009), a total of 22 children were referred to a muscle biopsy in suspicion of mitochondrial disorder based on the preliminary biochemical, metabolic and instrumental investigations. Enzymatic and/or molecular analysis confirmed mitochondrial disease in 5 of them - an SCO2 gene (synthesis of cytochrome c oxidase, subunit 2) defect, 2 cases of pyruvate dehydrogenase complex deficiency and 2 cases of combined complex I and IV deficiency. The live-birth prevalence for mitochondrial defects observed in our cohort was
\end{abstract}

$1 / 20,764$ live births. Our epidemiological data correlate well with previously published epidemiology data on mitochondrial diseases in childhood from Sweden and Australia, but are lower than in Finland.

Copyright $\odot 2012$ S. Karger AG, Basel

Mitochondrial disorders are a heterogeneous group of disorders affecting energy production of the body. They may present at any age with a spectrum of symptoms and signs to several medical specialties [Munnich and Rustin, 2001]. As cells with high-energy requirements, such as neurons, skeletal and cardiac muscle, are particularly vulnerable to limited adenosine triphosphate supply, encephalopathy and myopathy are often prominent features in the various mitochondrial phenotypes [Darin et al., 2001; Gropman, 2001; Kisler et al., 2010]. In addition, short stature, neurosensory hearing loss, progressive external ophtalmoplegia, axonal neuropathy, diabetes mellitus, and renal tubular acidosis are important symptoms of respiratory chain dysfunction [Darin et al., 2001].

Enzymes involved in the mitochondrial energy production are coded by $2-$ mitochondrial and nuclear - genomes in the organism. It is known that mitochondrial disorders in children result more often from nuclear DNA mutations. Lamont et al. [1998] demonstrated that

\section{KARGER}

Fax +4161306 1234

E-Mail karger@karger.ch

www.karger.com
(C) 2012 S. Karger AG, Basel

1661-8769/12/0033-0113\$38.00/0

Accessible online at:

www.karger.com/msy
Katrin Õunap

Department of Genetics, United Laboratories

Tartu University Hospital

2 L. Puusepa Street, EE-Tartu 51014 (Estonia)

Tel. +372 731 9490, E-Mail katrin.ounap@kliinikum.ee 
mitochondrial DNA mutations account for $<10 \%$ of all mitochondrial disorders in childhood. However, in Finnish children the frequency of pathogenic mitochondrial DNA mutations was found to be $18 \%$ [Uusimaa et al., 2004]. To date $>100$ point mutations in mitochondrial DNA are known [Finsterer, 2004], and >100 genes in nuclear DNA are known to affect energy production by oxidative phosphorylation system [Wong, 2010].

Due to clinical and genetic complexity, mitochondrial disorders represent a challenge to clinicians, especially in children, who show enormous variation in clinical presentation and course [Munnich and Rustin, 2001; Wolf and Smeitink, 2002; Morava et al., 2006; Honzik et al., 2012]. Although mitochondrial disorders are diagnosed more frequently, and the estimated prevalence in the general population is as high as $1 / 5,000$ [Skladal et al., 2003; Schaefer et al., 2004; Elliott et al., 2008], their clinical diagnostics is not straightforward. Due to the extremely nonspecific clinical picture, additional indications suggestive to mitochondrial disorder, including characteristic laboratory, metabolic and morphological abnormalities, are helpful in diagnosing. Screening based on measurement of lactate levels in serum and cerebrospinal fluid is used for first-line diagnostics, however, lactic acidosis may be present only in $50 \%$ of patients [Gropman, 2001; Koenig, 2008], up to $87 \%$ of patients with neonatal onset of mitochondrial disease [Honzik et al., 2012]. In patients with neurologic symptoms, cerebrospinal fluid lactate is considered to be more reliable than venous lactate [Koenig, 2008]. Elevated alanine in plasma, abnormal lactate/pyruvate ratio and elevated Krebs cycle intermediates observed in urinary organic acid analysis are the most widely observed metabolic changes [Gropman, 2001]. It was recently reported that the measurement of fibroblast growth factor 21 concentrations in serum identified primary muscle-manifesting respiratory chain deficiencies in adults and children and might be feasible as a first-line diagnostic test for these disorders [Suomalainen et al., 2011]. Most important instrumental investigations in patients with a suspected mitochondrial disease are brain magnetic resonance imaging (MRI) and spectroscopy. MRI is especially useful in children with nonspecific neurological symptoms and subtle biochemical and morphological abnormalities [Gropman, 2001; Kisler et al., 2010]. The most specific diagnostic information for mitochondrial disorders is obtained from examinations performed on muscle tissue. Biopsy specimens should be examined with routine light microscopy for structural changes, evaluated by histochemical, immunohistochemical and ultrastructural investigations. Biochemical analysis of respiratory chain enzymes can be performed on muscle biopsies, cultured skin fibroblasts or other tissues if necessary [Finsterer, 2004; Kisler et al., 2010].

Different consensus diagnostic criteria for mitochondrial disorders in childhood are available - Wolfson [Nissenkorn et al., 1999], Nijmegen [Wolf and Smeitink, 2002; Morava et al., 2006] and modified Walker criteria [Bernier et al., 2002]. They usually score major and minor criteria based on clinical, biochemical and molecular findings. Due to the extreme complexity of mitochondrial disorders in children, we decided to work out a diagnostic algorithm, applicable in clinical practice in Estonia, in order to identify patients with mitochondrial disorders among pediatric neurology patients and to evaluate the live-birth prevalence of mitochondrial disorders in childhood.

\section{Material and Methods}

\section{Definition of Study Group}

The study was performed from 2003 to 2009 in 2 regional hospitals (Tartu University Hospital and Tallinn Children's Hospital), which both serve as tertiary reference centers for entire Estonia. All patients referred to the departments of neonatology or pediatric neurology of those hospitals and were screened for the possible symptoms of mitochondrial disease.

\section{The Study Protocol}

The study protocol is schematically described in figure 1 . Serum lactate was routinely measured in all patients referred for investigation to the departments of pediatric neonatology or neurology of both hospitals. In case increased concentration was detected, lactate level was checked repeatedly to avoid elevations due to technical factors. If lactate level was constantly above reference range, mitochondrial disease was suspected, and metabolic and instrumental investigations were performed to establish the disease phenotype. If biochemical and instrumental investigations supported the diagnosis of mitochondrial disease, muscle biopsy was performed.

Pathomorphological studies, including immunohistochemical and ultrastructural examinations, were performed on muscle biopsy using routine histological evaluation, immunohistochemistry (including cytochrome c oxidase activity, staining for nicotinamide adenine dinucleotide and ragged red fibers) and electron-microscopy. In case changes characteristic to mitochondrial disease were found, biochemical investigations on muscle tissue were done to evaluate the function of respiratory chain enzyme complexes. If dysfunction of respiratory chain complexes was diagnosed in muscle tissue, their activity was also evaluated in skin fibroblasts in some cases. Finally, molecular studies were performed. The strategy for molecular investigations based on the clinical presentation, pathomorphological findings and biochemical phenotype observed in muscle tissue, and biochemical phenotype observed in skin fibroblasts. 
Fig. 1. Diagnostic algorithm for patients referred to muscle biopsy in suspicion of mitochondrial disease.

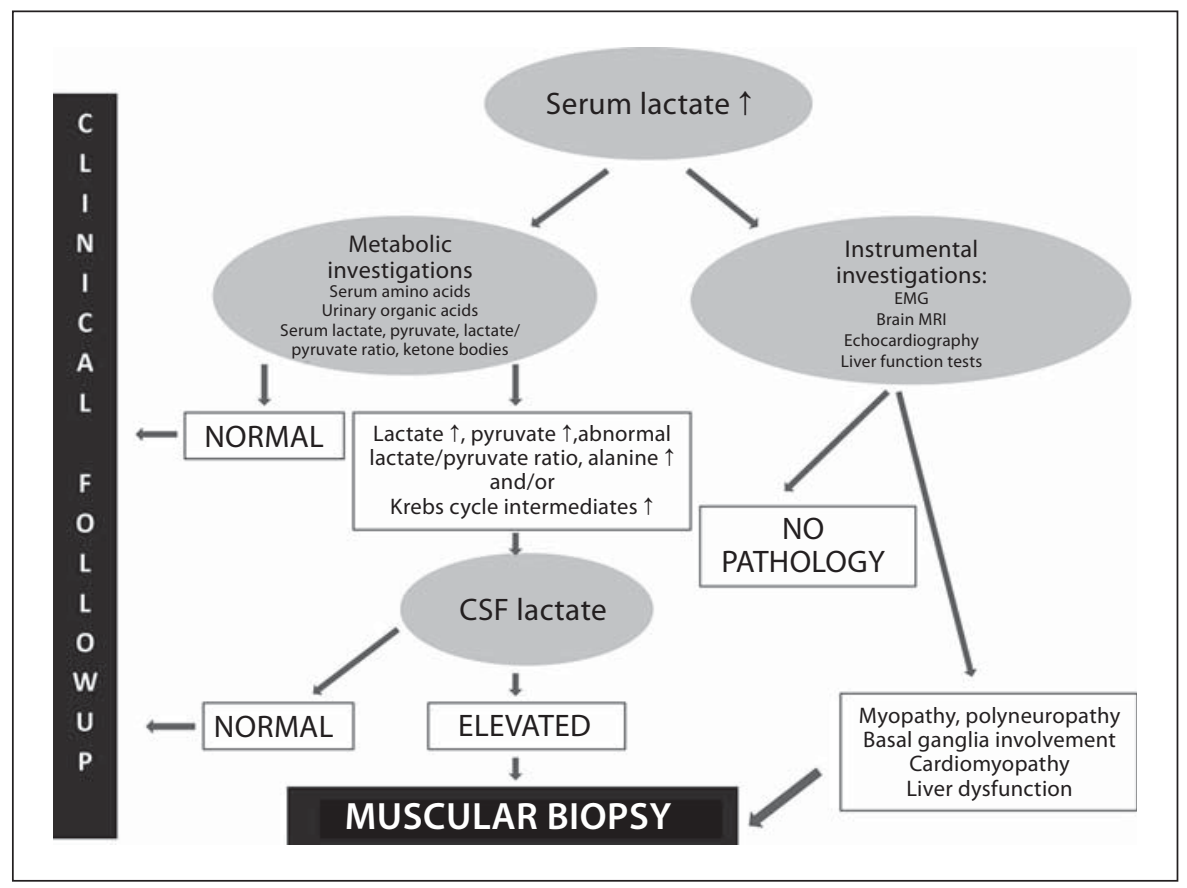

The live-birth prevalence of mitochondrial disorders was defined as the total number of cases with a mitochondrial defect born from 2003 to 2009, divided by the total number of live births in the same period. Annual live-birth data was obtained from the Statistical Database of the Statistics Estonia of the Ministry of Finance of Estonia (http://www.stat.ee). This study was approved by the Research Ethics Committee of the University of Tartu.

\section{Results}

In total, 22 children were referred to a muscle biopsy in suspicion of mitochondrial disorder based on the preliminary biochemical, metabolic and instrumental investigations. Pathomorphological changes characteristic to or suggestive of mitochondrial disease were found in 5 patients (table 1). Biochemical analysis performed from the muscle tissue confirmed mitochondrial disease in 3 of them: one (case 3) had complex IV deficiency caused by mutations in the SCO2 gene - synthesis of cytochrome c oxidase, subunit 2 formerly known as SCO cytochrome oxidase deficient homolog 2 (yeast) - [Joost et al., 2010], 2 patients (cases 1 and 2) had combined deficiency of complexes I and IV in muscle tissue (discussed below). Two of them (cases 4 and 5) had characteristic changes in muscle pathomorphology, but neither enzymatic nor molecular analyses could identify the exact etiology. Additionally, biochemical investigations performed on muscle tissue confirmed pyruvate dehydrogenase $(\mathrm{PDH})$ complex deficiency in 2 patients (cases 6 and 7), who had no pathomorphological changes suggestive of mitochondrial disease, but whose metabolic investigations were characteristic to PDH complex deficiency (not discussed here).

During the study period (2003-2009), 103,821 live births were recorded in Estonia. The live-birth prevalence for mitochondrial defects was $1 / 20,764$ live births.

\section{Case 1}

The girl was born in the third pregnancy and first delivery. Her birth weight was $4,190 \mathrm{~g}(+1.5 \mathrm{SD})$, length $52 \mathrm{~cm}(+1.5 \mathrm{SD})$ and Apgar score 7/8. During the first days of life, transient hypoglycemia and tachypnea were observed. At the age of 1 month, following the vaccination against tuberculosis and hepatitis B, progressive sluggishness, muscular hypotonia, feeding and respiratory difficulties were observed, and the child was hospitalized. Clinically severe muscular hypotonia and myopathic phenotype were observed. She had no active movements and needed artificial respiration using nasal continuous positive airway pressure as treatment. Serum lactate level was constantly elevated with a maximum of $9.1 \mathrm{~mm} / 1$ (reference <2.6). Electromyographic investigation (EMG) showed myopathic findings, whereas brain MRI showed no pathology. Cerebrospinal fluid (CSF) lactate was 3.67 $\mathrm{mM} / \mathrm{l}$ (reference 1.1-2.4). Amino acid analysis revealed increased concentration of alanine, $519.6 \mu \mathrm{M} / \mathrm{l}$ (reference 130-442), and elevated alanine/lysine ratio $\sim 10$ (reference $<3$ ). Urinary organic acid profile showed increased excretion of succinic, fumaric, glu- 

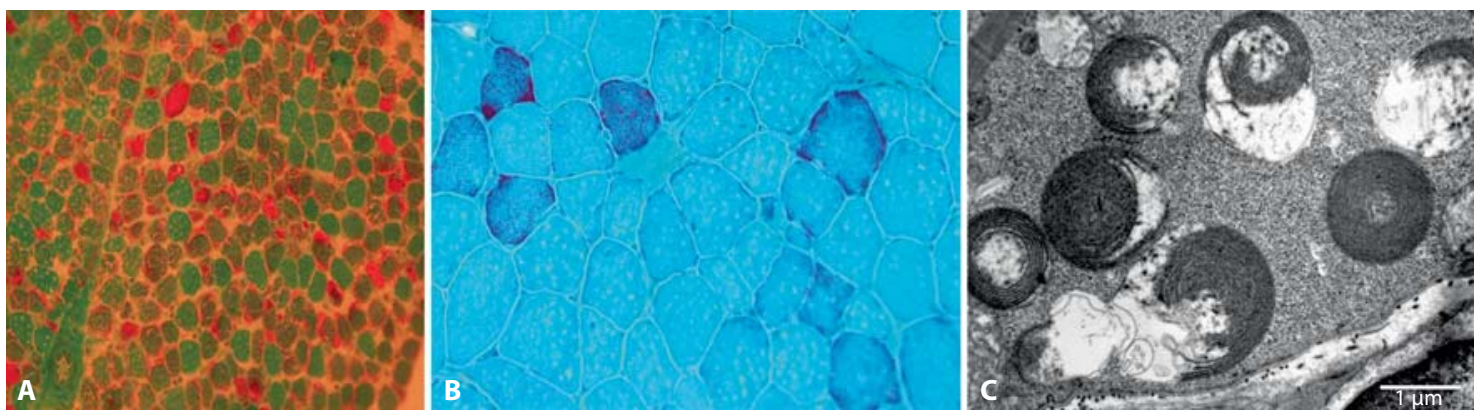

Fig. 2. Case 1 muscle biopsy. A Ragged red fibers at the age of 2 months. B At the age of 5 years. C Ultrastructure of pathological mitochondria (whorled mitochondria) in electron microscopic investigation [Joost et al., 2012].

Table 1. Pathomorphological findings, enzymatic activity of respiratory chain complexes and PDH complex, and molecular findings in the patients

\begin{tabular}{|c|c|c|c|c|c|c|c|}
\hline & $\begin{array}{l}\text { Age at } \\
\text { investigation }\end{array}$ & $\begin{array}{l}\text { Ragged } \\
\text { red fibers }\end{array}$ & $\begin{array}{l}\text { Cytochrome } \\
\text { c oxidase } \\
\text { activity }\end{array}$ & $\begin{array}{l}\text { Pathological } \\
\text { ultrastucture of } \\
\text { mitochondria }\end{array}$ & $\begin{array}{l}\text { Lipid } \\
\text { storages }\end{array}$ & $\begin{array}{l}\text { Enzymatic activity of } \\
\text { respiratory chain } \\
\text { complexes }\end{array}$ & Molecular analysis \\
\hline Case 1 & 1 month & + & ++ & +++ & +++ & $\begin{array}{l}\text { decreased activity of } \\
\text { complexes I and IV }\end{array}$ & mutation $\mathrm{m} .14674 \mathrm{~T}>\mathrm{C}$ in $\mathrm{mt}-\mathrm{RNA}^{\mathrm{Glu}}$ \\
\hline Case 2 & 1 month & - & not done & - & +++ & $\begin{array}{l}\text { decreased activity of } \\
\text { complexes I and IV }\end{array}$ & $\begin{array}{l}\text { no mutations in } P O L G \text { and } T K 2 \text { gene, } \\
\text { and in mitochondrial DNA }\end{array}$ \\
\hline $\begin{array}{l}\text { Case } 3 \\
\text { [Joost et al., } \\
\text { 2010] }\end{array}$ & 1 month & - & +++ & + & - & $\begin{array}{l}\text { decreased complex IV } \\
\text { activity }\end{array}$ & $\begin{array}{l}\text { c. } 418 \mathrm{G}>\mathrm{A} / \mathrm{c} .17 \mathrm{INS} 19 \mathrm{bp} \text { mutations in } \\
\text { SCO2 gene }\end{array}$ \\
\hline Case 4 & 15 years & - & - & +++ & - & normal & $\begin{array}{l}\text { no deletions/duplications and point } \\
\text { mutations }(\mathrm{m} .3243 \mathrm{~A}>\mathrm{G}, \mathrm{m} .8344 \mathrm{~A}>\mathrm{G} \\
\mathrm{m} .8993 \mathrm{~T}>\mathrm{G} / \mathrm{C} \text { ) in mitochondrial } \mathrm{DNA}\end{array}$ \\
\hline Case 5 & 15 years & ++ & - & ++ & - & normal & $\begin{array}{l}\text { no deletions/duplications and point } \\
\text { mutations }(\mathrm{m} .3243 \mathrm{~A}>\mathrm{G}, \mathrm{m} .8344 \mathrm{~A}>\mathrm{G} \text {, } \\
\text { m.8993T }>\mathrm{G} / \mathrm{C}) \text { in mitochondrial } \mathrm{DNA}\end{array}$ \\
\hline Case 6 & 1 month & - & - & - & - & $\begin{array}{l}\text { decreased activity of } \\
\text { PDH complex }\end{array}$ & c. $904 \mathrm{C}>\mathrm{T}$ mutation in the $P D H A 1$ gene \\
\hline Case 7 & 1 month & - & - & - & - & $\begin{array}{l}\text { decreased activity of } \\
\text { PDH complex }\end{array}$ & c. $904 \mathrm{C}>\mathrm{T}$ mutation in the PDHA1 gene \\
\hline
\end{tabular}

taric, and ketoglutaric acid. In both muscle biopsies (performed at the age of 2 months and again at the age of 5 years), pathological ultrastructure of mitochondria (whorled mitochondria) was the most prominent finding. At the age of 2 months, about $50 \%$ of fibers had ragged red fibers phenotype, and at the age of 5 years, about $5-6 \%$ of fibers were ragged red fibers (fig. 2). Many cytochrome $\mathrm{c}$ oxidase negative fibers were present in both biopsies. Biochemical investigations from muscle tissue revealed lowered activities of complexes I and IV (table 2). Enzymatic activities of respiratory chain complexes I-IV from fibroblasts were normal. Sequencing of $P O L G$ and DGOUK genes revealed no mutations. Mutation m.14674T $>$ C mt-tRNA ${ }^{\text {Glu }}$ was present in mitochondrial DNA.

\section{Clinical Course}

Supportive treatment for respiratory chain was initiated at the age of 1.5 months. Treatment schedule consisted of ubiquinone 20 $\mathrm{mg} / \mathrm{kg}$ daily, riboflavin $10 \mathrm{mg} / \mathrm{kg} /$ daily (max. $200 \mathrm{mg}$ daily) and L-carnitine $100 \mathrm{mg} / \mathrm{kg}$ daily. Then, $10-15 \%$ high-fat calories were added to her daily diet. First active movements occurred at the age of 4 months. At the age of 7 months, she was free of artificial respiration, and her physical activity started to improve gradually. She started to walk independently at the age of 1 year 10 months.

At the age of 7 years, she still requires the treatment supporting the respiratory chain function (child started to complain about muscle pain after discontinuation). Clinically she has muscular hypotonia and myopathic phenotype. She is slightly slower in physical activities than her age-matched mates, but her cognitive development is according to age. 
Table 2. Enzymatic activities of respiratory chain complexes measured from the muscle tissue of patients

\begin{tabular}{lllll}
\hline & $\begin{array}{l}\text { Complex I } \\
\text { mU/UCS }\end{array}$ & $\begin{array}{l}\text { Complex II+III } \\
\text { mU/UCS }\end{array}$ & $\begin{array}{l}\text { Complex IV } \\
\text { mU/UCS }\end{array}$ & $\begin{array}{l}\text { Citrate synthase } \\
\text { mU/mg }\end{array}$ \\
\hline Case 1 & 4 & 101 & 48 & 470 \\
Case 2 & 28 & 51 & 58 & 118 \\
Reference range & $84-559$ & $37-285$ & $520-2080$ & $45-187$ \\
\hline
\end{tabular}

UCS = Unit of citrate synthase.

\section{Case 2}

The girl was born in the third pregnancy and 2nd delivery. Her birth weight was 3,520 g (0 SD), length $50 \mathrm{~cm}(-1 \mathrm{SD})$ and Apgar score $8 / 9$. She developed breathing difficulties and hypoglycemia (s-glycose $2.4 \mathrm{~mm} / \mathrm{l}$; reference 2.8-4.4) on the third day of life. Additionally, she developed lactic acidosis up to $9.7 \mathrm{~mm} / 1$ (reference $<2.6)$. Clinically she had muscular hypotonia and a slightly dysmorphic phenotype with a broad forehead, hypoplastic supraorbital ridges and downturned corners of the mouth. EMG showed myopathic findings. Urinary organic acid analysis revealed increased excretion of Krebs cycle intermediates (lactic, 2-OH-butyric, fumaric, ketoglutaric, and 4-OH-phenyllactic acid). Amino acids from plasma were within the reference range, but urinary amino acid analysis revealed increased excretion of alanine. Lactate from CSF was $4.8 \mathrm{~mm} / \mathrm{l}$ (reference 1.1-2.4). Pathomorphological examination of muscle tissue showed lipid and glycogen storages in muscle fibers. No ragged red fibers were present. Cytochrome c oxidase activity could not be evaluated, due to technical reasons. Biochemical analysis of muscle tissue showed decreased activity of respiratory chain complexes I and IV (table 2). Respiratory chain activities from fibroblasts were normal. Sequencing of $P O L G$ and TK2 genes revealed no mutations. Also, no mutations were found in the mitochondrial DNA using Affimetrix Human Mitochondrial Resequencing Array 2.0.

\section{Clinical Course}

Due to breathing difficulties, the child was intubated at the age of 4 days and tracheostomy was established at the age of 2 weeks. Although her muscle strength improved gradually, she has remained dependent on artificial respiration and, therefore, is treated in the intensive care unit of the local hospital. At the age of 1 year, she had active movements in extremities, was able to grab toys, play with them, and turn to the side.

\section{Discussion}

Mitochondrial dysfunction may be one of the most common causes of the disease among children with unexplained encephalomyopathy, as $15 \%$ of such patients can be diagnosed with probable mitochondrial disease [Uusimaa et al., 2000]. The cardinal diagnostic procedure is muscle biopsy, which cannot be applied to all patients with clinical symptoms potentially linked to some form of respiratory chain disorder, due to invasiveness, timeconsumption and cost. Most of the available diagnostic schemes for mitochondrial disorders [Nissenkorn et al., 1999; Bernier et al., 2002; Wolf and Smeitink, 2002; Morava et al., 2006] are useful for evaluating the probability of mitochondrial disorders based on the pathomorphological and biochemical findings in the muscle tissue. Although most authors acknowledge the wide clinical spectrum of the diseases [Darin et al., 2001; Gropman, 2001; Kisler et al., 2010], published data is lacking clinical criteria in patients in whom muscle biopsy should be performed. Kisler et al. [2010] have published a review about the management of mitochondrial disorders in childhood covering all known clinical presentations, diagnostic strategy and handling. However, as the measurement of the respiratory chain enzymatic activity in tissue samples is not available in Estonia, careful preselection becomes utmost important for our clinical practice.

Therefore, we decided to work out a diagnostic algorithm for better identification of patients with possible mitochondrial disease among pediatric neurology patients, taking into account the possibilities of our local health care system. This algorithm relies on 2 presentations of those disorders: clinical presentation specified with instrumental investigations and biochemical phenotype. We find that this approach is easy to use in clinical practice as MRI and EMG investigations are routinely done in most patients with developmental delay and/or muscular hypotonia, and lactate measurement is available in most of the laboratories. Also, these preliminary investigations allow relatively quick clinical decisionmaking and treatment supporting mitochondrial function can be initiated earlier.

Mitochondrial disease was confirmed in 5 patients in our series. All patients had elevated serum and CSF lactate levels. In patients with PDH complex deficiency, high lactate and pyruvate level was the main clinical symptom initiating further investigations. All 3 confirmed patients 
Table 3. Prevalence of mitochondrial disorders in childhood in different populations

\begin{tabular}{llll}
\hline Study population & Prevalence & 95\% CI, per 100,000 & Reference \\
\hline Northern Finland children $<18$ years & $1: 5,634$ & not calculated & Uusimaa et al., 2000 \\
Western Sweden children $<16$ years & $1: 21,000$ & $2.8-7.6$ & Darin et al., 2001 \\
Victorian Australia children $<16$ years & $1: 20,000$ & $4.0-6.2$ & Skladal et al., 2003 \\
Estonia life-birth prevalence & $1: 20,764$ & $1.5-11.2$ & current study \\
\hline
\end{tabular}

with respiratory chain deficiency had elevated serum lactate with myopathic changes detected on EMG investigation. The patient with complex IV deficiency due to SCO2 deficiency additionally had radiological findings characteristic to Leigh syndrome. All patients had elevated excretion of Krebs cycle intermediates.

Another possibility to improve the diagnostic yield is to perform DNA analysis in all indications for muscle biopsy. Presently, it was not done due to high cost and workflow as mitochondriopathies arise due to mutations in many different genes of mitochondrial DNA or $>100$ nuclear DNA encoding for mitochondrial proteins with enzymatic, structural, signaling, channeling, transport, receptor, or assembling functions [Finsterer, 2004; Wong, 2010]. However, next generation sequencing has become the core technology for gene discovery in rare inherited disorders including mitochondrial disorders. Haack et al. [2012] recently showed that appropriate in silico filtering of exome sequencing data, coupled with functional validation of new disease alleles, is effective in rapidly identifying disease-causative variants in known and new complex I associated disease genes. This technology will be an alternative in the future.

The first epidemiological study evaluating mitochondrial abnormalities in children with unexplained psychomotor retardation was done in Finland, where a biochemical defect of oxidative phosphorylation was found in 1/5,634 children [Uusimaa et al., 2000]. Later epidemiological investigations have not succeeded in showing such high prevalence in Sweden [Darin et al., 2001] and Australia [Skladal et al., 2003]. Although Estonians are ethnically related to Finns, the live-birth prevalence observed in our cohort $(1: 20,764)$ correlates well with the Swedish and Australian data, supporting the conclusion that mitochondrial diseases have similar prevalence in different populations [Schaefer et al., 2004]. The comparison of epidemiological data is given in table 3 . One possible explanation for the difference between our and Finnish cohort might be the strict preselection criteria in our cohort - in the Finnish study a muscle biopsy was performed rou- tinely in patients with unexplained encephalomyopathy and myopathy. But this strategy is unreachable for us in daily clinical practice - therefore, the algorithm for clinical practice was developed.

As our algorithm bases on measurement of serum lactate level, the actual incidence of mitochondrial diseases in Estonia can be higher, since normal lactate level may be present in $30-70 \%$ of patients [Koenig, 2008]. In the future, more attention should probably be paid to the subtle biochemical changes (elevated excretion of Krebs cycle metabolites and alanine in case of normal lactate level etc.) and clinical presentation including the results of instrumental investigations. Fibroblast growth factor 21 measurement [Suomalainen et al., 2011] may also be considered in the future. All our patients with enzymatically confirmed mitochondrial disease presented in the newborn period, thus, it is possible to conclude that screening for mitochondrial disorders based on serum lactic acid measurement is informative in case of early-onset of the disease. It was recently shown that elevated level of lactate was found in $87 \%$ of patients with neonatal onset of mitochondrial disease [Honzik et al., 2012].

We identified 2 cases with combined deficiency of complexes I and IV in muscle tissue. In both cases, the treatment supporting mitochondrial function, consisting of coenzyme Q10, riboflavin and L-carnitine, together with low-carbohydrate and high-fat containing diet, was initiated as soon as preliminary suspicion of mitochondrial disease rose. The immediate response to the treatment was normalization of serum lactate level, first signs of clinical improvement occurred within some months. One of those patients (case 1) has cytochrome c oxidase deficiency due to homoplasmic m.14674T $>$ C mt-tRNA ${ }^{\text {Glu }}$ mutation identified as a cause of benign mitochondrial myopathy by Horvath et al. [2009]. Patients with homoplasmic m.14674T $>$ C mt-tRNA ${ }^{\text {Glu }}$ mutations present with clinical deterioration shortly after birth or within the first months of life, characterized clinically by lactic acidosis, profound hypotonia and feeding difficulties. Although remarkable recovery is observed in those patients, 
mild myopathy is present up to adulthood [Uusimaa et al., 2011]. This is the case with our patient as well. She showed gradual improvement within first 3 years of life, but at the age of 7 years, she still has myopathic phenotype, and her physical abilities are lower compared to coevals. The treatment supporting mitochondrial respiratory chain function is still necessary for her, as she starts to complain about muscle pain if the treatment is discontinued.

The molecular cause of the disease of case 2 with combined deficiency of complexes I and IV is still unknown. Although the patient showed some improvement during the first year: she grew accordingly, her muscle strength improved quickly and active movements developed, her improvement slowed down on the 2 nd year of life. Whether it is related to the genetic cause of her disease, or to the limitations of her physical activity due to artificial respiration, is still unclear.
In conclusion, we have developed an algorithm for diagnosing mitochondrial diseases, which takes into account our local diagnostic possibilities. Although our schedule has some shortcomings, as some cases with normal lactate level and subtle clinical symptoms can be missed in the early stages of the disease, those problems can be overcome conducting thorough clinical followups of the patients. This systematic approach has made the identification of patients with mitochondrial disease in our country possible, improved the treatment of patients and genetic counseling of the families.

\section{Acknowledgements}

We thank the families for participating in the study. This work was supported by Estonian Science Foundation grant GARLA 8175 .

\section{References}

Bernier FP, Boneh A, Dennett X, Chow CW, Cleary MA, Thorburn DR: Diagnostic criteria for respiratory chain disorders in adults and children. Neurology 59:1406-1411 (2002).

Darin N, Oldfors A, Moslemi AR, Holme E, Tulinius $\mathrm{M}$ : The incidence of mitochondrial encephalomyopathies in childhood: clinical features and morphological, biochemical, and DNA abnormalities. Ann Neurol 49: 377-383 (2001).

-Elliott HR, Samuels DC, Eden JA, Relton CL, Chinnery PF: Pathogenic mitochondrial DNA mutations are common in the general population. Am J Hum Genet 83:254-260 (2008).

Finsterer J: Mitochondriopathies. Eur J Neurol 11:163-186 (2004).

Gropman AL: Diagnosis and treatment of childhood mitochondrial diseases. Curr Neurol Neurosci Rep 1:185-194 (2001).

Haack TB, Haberberger B, Frisch EM, Wieland T, Iuso A, et al: Molecular diagnosis in mitochondrial complex I deficiency using exome sequencing. J Med Genet 49:277-283 (2012).

Honzik T, Tesarova M, Magner M, Mayr J, Jesina $\mathrm{P}$, et al: Neonatal onset of mitochondrial disorders in 129 patients: clinical and laboratory characteristics and a new approach to diagnosis. J Inherit Metab Dis 2012, E-pub ahead of print.

- Horvath R, Kemp JP, Tuppen HA, Hudson G, Oldfors A, et al: Molecular basis of infantile reversible cytochrome $\mathrm{c}$ oxidase deficiency myopathy. Brain 132:3165-3174 (2009).
Joost K, Rodenburg R, Piirsoo A, van den Heuvel B, Zordania R, Ounap K: A novel mutation in the $\mathrm{SCO} 2$ gene in a neonate with early-onset cardioencephalomyopathy. Pediatr Neurol 42:227-230 (2010).

Kisler JE, Whittaker RG, McFarland R: Mitochondrial diseases in childhood: a clinical approach to investigation and management. Dev Med Child Neurol 52:422-433 (2010).

Koenig MK: Presentation and diagnosis of mitochondrial disorders in children. Pediatr Neurol 38:305-313 (2008).

Lamont PJ, Surtees R, Woodward CE, Leonard JV, Wood NW, Harding AE: Clinical and laboratory findings in referrals for mitochondrial DNA analysis. Arch Dis Child 79: 22-27 (1998).

Morava E, van den Heuvel L, Hol F, de Vries MC, Hogeveen M, et al: Mitochondrial disease criteria: diagnostic applications in children. Neurology 67:1823-1826 (2006).

Munnich A, Rustin P: Clinical spectrum and diagnosis of mitochondrial disorders. Am J Med Genet 106:4-17 (2001).

Nissenkorn A, Zeharia A, Lev D, Fatal-Valevski A, Barash V, et al: Multiple presentation of mitochondrial disorders. Arch Dis Child 81: 209-214 (1999).

Schaefer AM, Taylor RW, Turnbull DM, Chinnery PF: The epidemiology of mitochondrial disorders - past, present and future. Biochim Biophys Acta 1659:115-120 (2004).
Skladal D, Halliday J, Thorburn DR: Minimum birth prevalence of mitochondrial respiratory chain disorders in children. Brain 126: 1905-1912 (2003).

-Suomalainen A, Elo JM, Pietilainen KH, Hakonen AH, Sevastianova K, et al: FGF-21 as a biomarker for muscle-manifesting mitochondrial respiratory chain deficiencies: a diagnostic study. Lancet Neurol 10:806-818 (2011).

- Uusimaa J, Remes AM, Rantala H, Vainionpää L, Herva R, et al: Childhood encephalopathies and myopathies: a prospective study in a defined population to assess the frequency of mitochondrial disorders. Pediatrics 105: 598-603 (2000).

-Uusimaa J, Finnilä S, Remes AM, Rantala H, Vainionpää L, et al: Molecular epidemiology of childhood mitochondrial encephalomyopathies in a Finnish population: sequence analysis of entire mtDNA of 17 children reveals heteroplasmic mutations in tRNA ${ }^{\mathrm{Arg}}$, tRNA $^{\text {Glu }}$, and tRNA ${ }^{\text {Leu(UUR })}$ genes. Pediatrics 114:443-450 (2004).

Uusimaa J, Jungbluth H, Fratter C, Crisponi G, Feng L, et al: Reversible infantile respiratory chain deficiency is a unique, genetically heterogenous mitochondrial disease. J Med Genet 48:660-668 (2011).

-Wolf NI, Smeitink JA: Mitochondrial disorders: a proposal for consensus diagnostic criteria in infants and children. Neurology 59:14021405 (2002).

-Wong LJ: Molecular genetics of mitochondrial disorders. Dev Disabil Res Rev 16:154-162 (2010). 